\title{
Deportes colectivos universitarios y su incidencia en el rendimiento académico en la Escuela Superior Naval.
}

\section{Collective university sports and its impact on academic performance at the Naval High School.}

Welinton Cristóbal Merino Narváez. ${ }^{1}$, Monge Sánchez Mauricio Javier. ${ }^{2}$ \& Asdrúbal Bayardo Merino Narváez. ${ }^{3}$ \& Luis Alfonso Balseca Aulestia. ${ }^{4}$

\section{Abstract. \\ DOI: https://doi.org/10.33262/cienciadigital.v3i2.449}

The research to be carried out bases its importance on the in-depth analysis of the collective sports with greater incidence and the academic performance of the students of the Naval Superior School. The causes that produce a poor academic performance of athletes when forming a selection are many, but among the most common are: the lack of study habits, academic irresponsibility, tardiness in the presentation of tasks, consecutive absences to classes of subjects with a greater degree of difficulty. Academic neglect produces a large amount of repetition and student desertion, all these results are exposed and documented in the tabulations made of the surveys.

Keywords: Collective Sports, Academic Performance.

\section{Resumen.}

La investigación a realizarse basa su importancia en el análisis profundo de los deportes colectivos con mayor incidencia y el rendimiento académico de los estudiantes de la Escuela Superior Naval. Las causas que producen un deficiente rendimiento académico de los deportistas al conformar una selección son muchas, pero entre las más comunes están: el no tener hábitos de estudio, irresponsabilidad académica, impuntualidad en la

\footnotetext{
1 Universidad de las Fuerzas Armadas, Salinas, Ecuador.wcmerino@espe.edu.ec

2 Unidad Educativa Municipal del Milenio Bicentenario, Quito, Ecuador, chino8javier@gmail.com

3 Institución Educativa Juan Pio Montufar, Quito, Ecuador, asdru123@ hotmail.com

4 Institución Educativo Fiscal Quito, Quito, Ecuador, luisiardy@ hotmail.com
} 
presentación de tareas, faltas consecutivas a clases de asignaturas con un mayor grado de dificultad. El descuido académico produce gran cantidad de repitencia y deserción estudiantil, todos estos resultados son expuestos y documentados en las tabulaciones realizadas de las encuestas.

Palabras claves: Deportes Colectivos, Rendimiento Académico.

\section{Introducción.}

Los estudiantes practican deportes colectivos, como: futbol, básquet, vóley entre otros, los cuales inciden en el rendimiento académico de la Escuela Superior Naval de una manera negativa, en especial de aquellos estudiantes que practican actividad deportiva extracurricular.

Thorpe (1992) vio que muchos/as de los/as que acababan la etapa educativa tenían poco conocimiento acerca del deporte; los/as más habilidosos/as no tomaban buenas decisiones teniendo una limitación técnica y dependían en gran medida del profesorado. (como se cita en Nuñoz R. 2014)

Se demuestra que los estudiantes que practican actividad deportiva diaria en una forma sustancial, obtienen beneficios como; mejor funcionamiento del cerebro en términos cognitivos, niveles más altos de concentración de energía, cambios en el cuerpo que mejoran la autoestima y un mejor comportamiento que incide sobre los procesos de enseñanzaaprendizaje (Bouchard, Malina, \& Pérusse, 1997; Silliker \& Quirk, 1997; Taras, 2005).

Los autores (Bouchard et al., 1997) destacan que la práctica de actividad física incrementa los niveles de fuerza muscular, de flexibilidad, equilibrio y de coordinación en los jóvenes. Este aspecto beneficia la disminución del riesgo de lesiones en la edad adulta. La presente contribución muestra una revisión bibliográfica de los artículos más consultados en la temática de la actividad física y su relación con el desempeño académico

Siendo textuales en los beneficios comprobados de practicar deportes, los estudiantes que realizan deportes colectivos en la Escuela Superior Naval, deberían presentar un mejor desempeño en sus labores académicas, lastimosamente los datos tabulados muestran todo lo contrario. Aquellos estudiantes que forman parte de la selección deportiva y practican deportes como opción extracurricular, muestran un serio descenso en sus calificaciones académicas.

En el siglo XXI la gran mayoría de estudiantes universitarios llevan una vida sedentaria y carecen de destrezas para organizar su tiempo y tareas, que les permita practicar un deporte colectivo sin descuidar la parte académica. Las causas más importantes son, el no tener hábitos de estudio, irresponsabilidad académica, impuntualidad en la presentación de tareas, faltas consecutivas a clases de asignaturas con un mayor grado de dificultad 
El presente trabajo de investigación, pretende encontrar los problemas más comunes, que aquejan a los estudiantes universitarios de la Escuela Superior Naval, que practican deportes colectivos y presentan una disminución en su rendimiento académico.

Por otra parte, la pedagogía tradicional de los docentes, la falta de motivación, la falta de un tiempo para realizar una recuperación pedagógica de los contenidos de las materias con mayor dificultad, estos problemas hacen que el deportista de elite tenga una estadística del 15\% de deserción estudiantil y un 30\% de pérdida del año, bien, no es un parámetro que pueda medir en todos los jóvenes que practican deporte o participan actividad física semanalmente en forma activa, que esta sea un factor determinante de mejora en el rendimiento académico. $\mathrm{Si}$, es posible constatar que estos jóvenes son indudablemente más activos e inquietos, pueden responder a múltiples estímulos a la vez sin perder la capacidad de concentración, (como se cita en W.Merino 2012) "el deporte, en sus manifestaciones como práctica para todos y como Deporte de Competencia, contribuye de manera esencial al desarrollo integral del ser humano. Asimismo, la salud, agente indispensable en el bienestar del individuo y parte integrante de la calidad de vida, es, sin duda, uno de los grandes favorecidos de la práctica deportiva"(como se cita en W.Merino 2012)

\section{Metodologia.}

Según Naresh K. Malhotra, las encuestas son entrevistas con un gran número de personas utilizando un cuestionario prediseñado. Según el mencionado autor, el método de encuesta incluye un cuestionario estructurado que se da a los encuestados y que está diseñado para obtener información específica

La metodología propuesta se basa en la investigación de campo aplicando una encuesta a los 150 deportistas de la Escuela Superior Naval.

\section{Modelo de la encuesta.}

Los tipos de encuestas según los fines científicos, el objetivo principal de la investigación, exploratorias, descriptivas, Explicativas, predictiva, evaluativa, los tipos utilizados en la investigación son descriptiva y evaluativa.

¿Obtiene bajo rendimiento académico al practicar deportes colectivos?

$\begin{array}{ll}\text { Si } & \text { ( ) } \\ \text { No } & \text { ( ) }\end{array}$


¿Por qué cree que es positivo practicar deportes colectivos?

Por salud

( )

Sociabilidad

$(1$

Mejor rendimiento intelectual

Concentración intelectual

¿Cuál de estas dos opciones considera prioritaria para practicar deportes colectivos?

Utilización de los implementos necesarios que eviten lesiones （ ）

Antes de practicar su deporte realizar un precalentamiento. （ )

¿Qué tipos de deportes colectivos son los más practicados?
Futbol
( )
básquet
( )
Vóley
( )

\section{Resultados}

Mediante la aplicación de la encuesta se obtuvieron los siguientes resultados.

Tabla No 1. Obtiene un bajo rendimiento académico al practicar deportes colectivos

\begin{tabular}{lll}
\hline Pregunta No 1 & Numero estudiantes & Porcentaje \\
\hline $\mathrm{Si}$ & 125 & $85 \%$ \\
$\mathrm{No}$ & 25 & $15 \%$ \\
\hline
\end{tabular}

Elaborado por: Grupo de Investigación 
Gráfico No1: Rendimiento académico

\section{Obtiene bajo rendimiento academico al practicar} deportes colectivos?

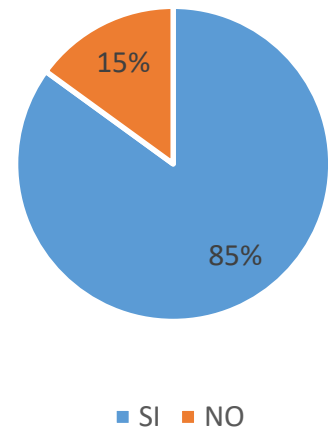

Elaborado por: Grupo de Investigación

Análisis: El 85\% de los estudiantes encuestados manifiestan que obtiene bajo rendimiento académico al practicar deportes colectivos y el 15\% de estudiantes no mejorar, por lo que se concluye que la mayoría de estudiantes ha tenido un bajo rendimiento académico al practicar deportes colectivos.

Tabla No 2. Por qué cree que es positivo practicar deportes colectivos

\begin{tabular}{lll}
\hline Pregunta No 1 & Numero estudiantes & Porcentaje \\
\hline Por salud & 75 & $50 \%$ \\
Por sociabilidad & 25 & $16.7 \%$ \\
Rendimiento intelectual & 24 & $16 \%$ \\
Concentración intelectual & 26 & $17.3 \%$ \\
\hline
\end{tabular}

Elaborado por: Grupo de Investigación 
Gráfico No2: Practica deportiva

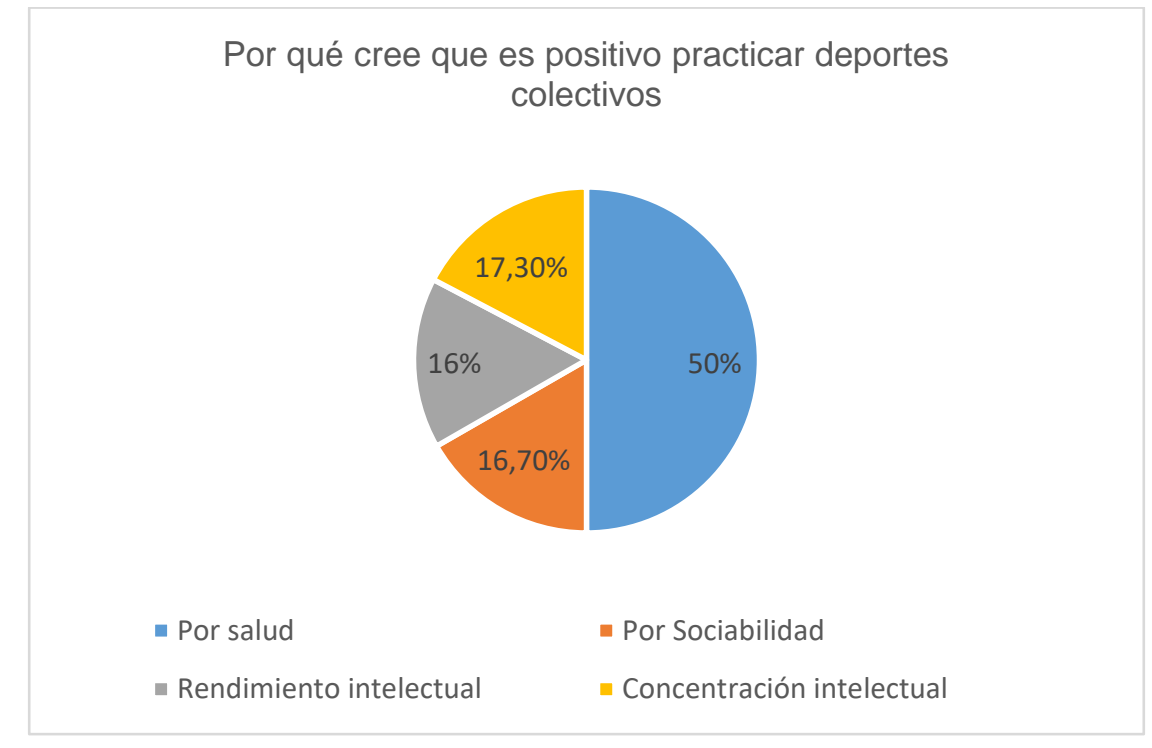

Elaborado por: Grupo de Investigación

Análisis: El 50\% de los estudiantes encuestados practica deporte por salud, el 17,30\% por rendimiento intelectual, el $16.70 \%$ por sociabilidad, el $16 \%$ rendimiento Intelectual al practicar deportes colectivos, por lo que se concluye que la mayoría de estudiantes ha tenido un mejoramiento intelectual y físico de su cuerpo.

Tabla No 3. Cuál de estas dos opciones considera prioritaria para practicar deportes colectivos.

\begin{tabular}{lcc}
\hline Pregunta No 1 & Numero estudiantes & Porcentaje \\
\hline $\begin{array}{l}\text { Utilización de los } \\
\text { implementos necesarios }\end{array}$ & 60 & $40 \%$ \\
$\begin{array}{l}\text { que eviten lesiones } \\
\begin{array}{l}\text { Antes de practicar su } \\
\text { deporte realizar un } \\
\text { precalentamiento }\end{array}\end{array}$ & 90 & $60 \%$ \\
\hline
\end{tabular}

Elaborado por: Grupo de Investigación 
Gráfico No3: Práctica deportiva colectivas

\section{Cuál de estas dos opciones considera prioritaria para} practicar deportes colectivos

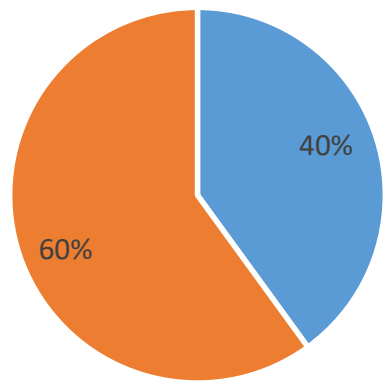

- Utilización de los implementos necesarios que eviten lesiones

- Antes de practicar su deporte realizar un precalentamiento

Elaborado por: Grupo de Investigación

Análisis: el $60 \%$ realiza un calentamiento antes de practicar deporte colectivo. Se concluye que es muy importante el calentamiento en los deportes colectivos. Se concluye que es muy importante realizar un calentamiento antes realizar los deportes colectivos. Para obtener mejores resultados físico y social.

Tabla No 4. Cree usted que practicar deportes colectivos disminuye el stress estudiantil

\begin{tabular}{lll}
\hline Pregunta No 1 & Numero estudiantes & Porcentaje \\
\hline Si & 135 & $90 \%$ \\
No & 15 & $10 \%$ \\
\hline
\end{tabular}

Elaborado por: Grupo de Investigación 
Gráfico No4: Práctica deportiva colectiva

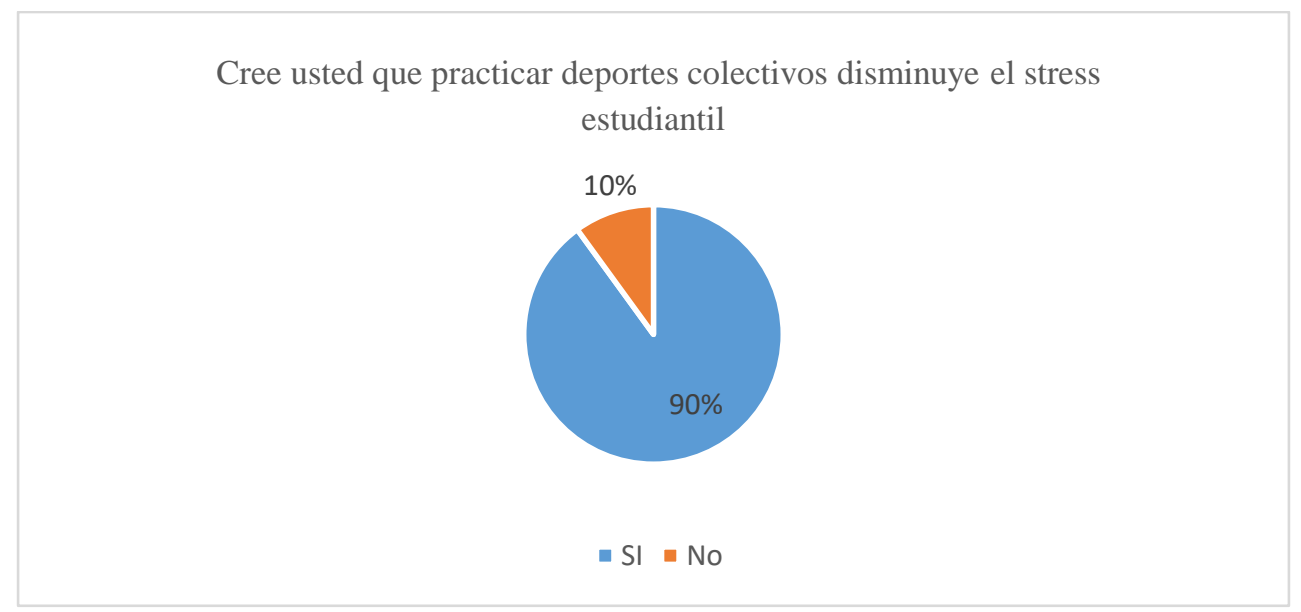

Elaborado por: Grupo de Investigación

Análisis: El 90\% de los estudiantes encuestados manifiesta que practicar deportes colectivos disminuye el stress estudiantil, mientras que el 10\% menciona que no ayuda, Concluimos que practicar deportes colectivos reduce los niveles de estrés causados por el estudio. Por lo tanto, el estudiante obtiene un mejor rendimiento académico

La mayor cantidad de profesores se ubica en el área de Ciencias Navales y Logística Naval. Las técnicas de estudio aplicadas al rendimiento académico de los guardiamarinas tienen una compresión del conocimiento en $85 \%$ según las encuestas realizadas a los estudiantes y docentes de la institución

Considero que los deportes colectivos más practicados y con resultados de aporte el en aprendizaje de un deportista seleccionados son.

Los estudiantes manifiestan que practicar deportes colectivos les ayuda a obtener una autoestima eleva en un $85 \%$, por ende, a reducir la depresión y superar cualquier inconveniente académico y social.

El futbol en un $75 \%$ es practicado por los estudiantes universitarios, básquet en un $20 \%$ y el Vóley en un 5\% y otros deportes en un 5\%.

Los entrenadores deportivos no se preocupan por los resultados académicos de los estudiantes deportistas 
Los padres de familia no ayudan al control del rendimiento académico de los estudiantes deportistas de la escuela por motivo de tiempo.

\section{Conclusiones.}

- Se determina que el $49 \%$ de estudiantes en el entorno familiar no recibe amor y comprensión; el 39\% de padres y madres no demuestran una actitud apropiada cuando aplican normas de disciplina y corrección; el 51\% presenta una escasa comunicación que aumenta la desconfianza y desmotivación en el aprendizaje cognitivo.

- Se establece que los padres de familia en un $36 \%$ no asiste ni participa en el desarrollo del aprendizaje cognitivo de sus hijos; además el $31 \%$ de educandos no recibe orientación en la revisión de tareas escolares influyendo en el conocimiento y hábitos de estudio; un 30\% de progenitores no ayudan en el refuerzo de temas académicos vistos en el aula; un $65 \%$ exterioriza dificultades la percepción, memoria, lenguaje y razonamiento, siendo una debilidad en la comprensión, la interpretación de la realidad y adquisición de experiencias.

- Se identifica que los estudiantes en el entorno familiar no reciben afectividad, comprensión, apoyo en la labor educativa, elementos influyentes en el aprendizaje cognitivo que al no ser fortalecidos perjudica en el desarrollo de competencias, la inteligencia, la toma de decisiones y resolución de problemas, por las razones indicadas es necesario sistematizar los resultados de la investigación realizada.

\section{Referencias bibliográficas.}

(Muñoz, 2014, pág. 24)la evaluación del aprendizaje de los deportes colectivos en educación física a través del modelo de enseñanza comprensivo.

(Merino, 2012, págs. 12,27) actividad deportiva extracurricular en el rendimiento académico

(Malhotra, 2016) https://www.promonegocios.net/mercadotecnia/encuestasdefinicion.html

(Pivarnik, 2006)Genetic and energy balance interactions in humans. In: Nutrients and Gene Expression: Clinical Aspects (Berdanier,

(http://pasalsoto.blogspot.com/2015/12/41-familia.html)

(Pivarnik, 2006)http://www.doctorperu.com/articulo-324-Mas-ejercicio-fisico-igualmejor-rendimiento-escolar.phpz 


\section{PARA CITAR EL ARTÍCULO INDEXADO.}

Merino W., Sánchez M., Bayardo A. \& Balseca L. (2019). Deportes colectivos universitarios y su incidencia en el rendimiento académico en la Escuela Superior Naval. Revista electrónica Ciencia Digital 3(2), 519-528. Recuperado desde: http://cienciadigital.org/revistacienciadigital2/index.php/CienciaDigital/article/view/449/1027

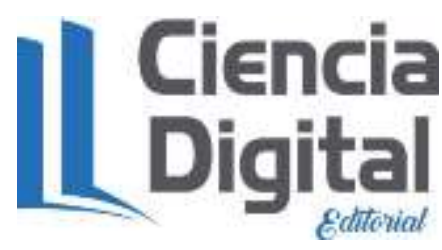

El artículo que se publica es de exclusiva responsabilidad de los autores y no necesariamente reflejan el pensamiento de la Revista Ciencia Digital.

El artículo queda en propiedad de la revista y, por tanto, su publicación parcial y/o total en otro medio tiene que ser autorizado por el director de la Revista Ciencia Digital.
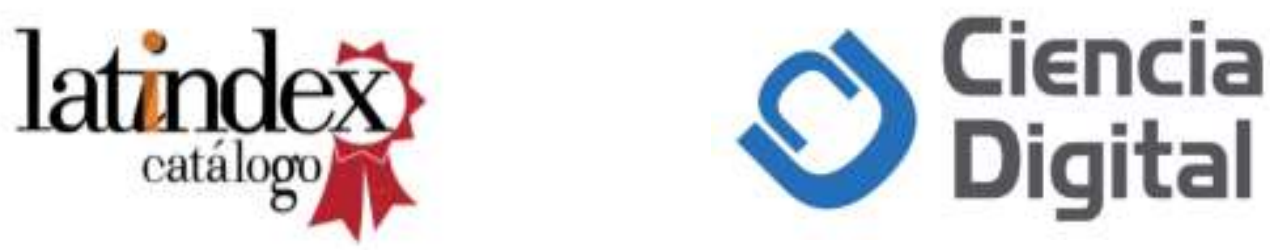